\title{
Modeling and Simulation of Long-Wave Infrared InAs/GaSb Strained Layer Superlattice Photodiodes with Different Passivants
}

\author{
Koushik Banerjee ${ }^{1, *}, J^{\prime}$ Huang ${ }^{1}$, Siddhartha Ghosh ${ }^{1}$ \\ ${ }^{1}$ Photonics and Magnetics Laboratory (ECE Department), University of Illinois at Chicago, \\ 851 S Morgan St, Room SEO 1020, Chicago, IL, 60607 \\ * koushik.banerjee@gmail.com, Ph\# 312-996-4184, fax\# 312-996-6465
}

\begin{abstract}
Current-voltage characteristics of long-wave infrared (LWIR) InAs/GaSb strained layer superlattice photodiodes (cut-off wavelength $\sim 10 \mu \mathrm{m}$ ), passivated with different surface passivants, have been modeled and simulated using ATLAS software from SILVACO. The simulated results are fitted to previous experimental results obtained on unpassivated devices and those passivated by silicon-dioxide $\left(\mathrm{SiO}_{2}\right)$, silicon nitride $\left(\mathrm{Si}_{\mathrm{x}} \mathrm{N}_{\mathrm{y}}\right)$ and zinc sulfide $(\mathrm{ZnS})$. Surface parameters in terms of surface recombination velocity, shunt resistance and interface trap density are extracted for different passivants. The performance of silicon-dioxide passivated diode is solely dominated by a shunt leakage path with a shunt resistance value of $0.56 \Omega-\mathrm{cm}^{2}$. Extracted electron and hole surface recombination velocities have values of $10^{5} \mathrm{~cm} / \mathrm{s}$ and $10^{7} \mathrm{~cm} / \mathrm{s}$ for unpassivated, $10^{3} \mathrm{~cm} / \mathrm{s}$ and $10^{5} \mathrm{~cm} / \mathrm{s}$ for $\mathrm{Si}_{\mathrm{x}} \mathrm{N}_{\mathrm{y}}$ passivated and $10^{2} \mathrm{~cm} / \mathrm{s}$ and $10^{3} \mathrm{~cm} / \mathrm{s}$ for $\mathrm{ZnS}$ passivated devices. Interface trap density follows a similar trend with values of $10^{15} \mathrm{~cm}^{-2}, 8.5 \times 10^{14} \mathrm{~cm}^{-2}$ and $10^{10} \mathrm{~cm}^{-2}$ for unpassivated, $S i_{x} N_{y}$ passivated and $\mathrm{ZnS}$ passivated devices respectively. The suitability and limitations of the simulation tool are discussed.
\end{abstract}

Keywords: InAs/GaSb, strained layer superlattice, passivation, long wavelength infrared, silvaco ATLAS, simulation

\section{Introduction}

Since its inception [1] as a material for infrared (IR) detection, indium arsenide (InAs) and gallium antimonide ( $\mathrm{GaSb}$ ) based strained layer superlattice (SLS) material 
system has matured with superior growth techniques using molecular beam epitaxy (MBE). It can be grown uniformly on comparatively large $\mathrm{GaSb}$ wafers with precise control of the SLS structures. It has a higher electron effective mass rendering lower tunneling currents. Moreover flexibility of SLS structure design permits band structure engineering to suppress Auger recombination [2] and enhance single carrier dominated impact ionization $[3,4]$. Owing to superior growth capabilities, comparable absorption coefficients [5] and possibility of better carrier dynamics, it has come up as an alternative to mercury cadmium telluride (HgCdTe) for detection of mid-wave and long-wave infrared (MWIR and LWIR) wavelengths.

Accurate simulation of semiconductor devices is an important requirement for the development process. Simulations can expedite design and optimization of the device structure without actually fabricating each possible option. Moreover, the simulation results can be useful in elucidating underlying physical mechanisms and predicting device performance parameters which are not readily measurable. Silvaco ATLAS is a commercially available device simulation framework that has been effectively used for the simulation of various types of devices based on traditional materials [6-11]. However, it provides little built-in support for emerging material like SLS due to a lack of proper understanding and availability of associated physical models and parameters. In this work, an attempt is made to model and simulate the current-voltage characteristics of differently passivated LWIR SLS diodes using ATLAS and extract surface related physical parameters through fitting of simulated results to those obtained experimentally in reference [12]. Surface leakage currents have been a major impediment to successful implementation of the SLS material system. So it is not sufficient to simulate only the bulk mechanisms and absolutely important to include and quantify the surface effects for the simulation of SLS diodes. Several research studies have been conducted on SLS passivation; however, this particular study carried out a comparative study on different passivants and provides a suitable reference for simulating bulk and surface properties of LWIR SLS.

\section{Device Structure and Simulation Models}

The original device had a $\mathrm{P}^{+}-\mathrm{n}^{-}-\mathrm{N}^{+}$structure grown on GaSb. The $1.9 \mu \mathrm{m}$ thick lightly doped LWIR region, 300 periods of 13 monolayers (MLs) InAs/ 7 MLs GaSb/ 1.3 MLs InSb unintentionally doped to $4 \times 10^{16} \mathrm{~cm}^{-3}$ n-type, was grown on a $350 \mathrm{~nm} \mathrm{MWIR} \mathrm{N}\left(4 \times 10^{18} \mathrm{~cm}^{-}\right.$ $\left.{ }^{3}\right)$ region. It was capped by a $230 \mathrm{~nm} \mathrm{P}^{+}\left(2 \times 10^{18} \mathrm{~cm}^{-3}\right)$ MWIR region. MWIR regions comprised of $8 \mathrm{MLs} \mathrm{GaSb} / 8 \mathrm{MLs}$ InAs. The thickness dimensions and dopings of the simulated device structure were exactly the same as that used in the experiment. The simulated structures had a $1 \mu \mathrm{m} \times 1 \mu \mathrm{m}$ cross-section unlike circular cross-section of diameter $400 \mu \mathrm{m}$ in the experimental devices. Simulating the exact structure will require impractically high runtimes and hence could not be pursued. The iterative algorithm failed to converge while simulating the $\mathrm{n}-\mathrm{N}$ heterojunction as present in the actual device. Including thermionic emission model, which might be the principal mechanism of carrier transport at such a junction, rendered absurdly high current outputs and hence was discarded. The 
heterojunction was replaced with a homojunction to circumvent the situation. The P-n heterojunction, which primarily dictates the device operation, was simulated exactly as in the experiment. The simulated structure is depicted in figure 1. Since ATLAS cannot directly simulate the SLS structures, the material is defined as a bulk in the input deck instead of a periodic arrangement of thin layers. Proper parameter values are selected to simulate its properties accurately.

The simulation framework uses the BLAZE platform dedicated to handle III-V materials. Different current mechanisms, which affect the operation of low bandgap diodes, are included by proper choice of available semiconductor models [13]. The drift-diffusion model is solved by default using equation (1),

$$
\vec{J}_{n, \psi}=q\left(n_{s} p\right) \mu_{n, \vec{E}} \pm q D_{n, \psi} \nabla n_{w} p
$$

where $J$ represents current density, $q$ electronic charge, $\mu$ mobility, $E$ electric field, $D$ diffusion constant and $n, p$ electron and hole concentration. The subscripts $n$ and $p$ are used to denote electron and hole components.

The generation-recombination currents are controlled by specifying radiative recombination rate $\left(C_{\text {opt }}\right)$ for optical (band-to-band) transitions, Auger recombination rates $\left(A_{n, p}\right)$ for three carrier recombination and carrier lifetimes $\left(\tau_{\mathrm{n}, \mathrm{p}}\right)$ associated with ShockleyReed-Hall (SRH) mechanism involving traps. The direct band to band transitions are modeled using equation (2),

$$
R_{\text {opt }}=\complement_{\text {ogt }}\left(n p-n_{i}^{2}\right)
$$

where $R_{\text {opt }}$ is the net optical recombination rate and $n_{i}$ is the intrinsic carrier density which is a function of semiconductor bandgap and temperature. Net Auger recombination is modeled as,

$$
E_{\text {Awger }}=A_{n}\left(p n^{2}-m m^{2}\right)+A_{g}\left(n p^{2}-q n_{n}^{2}\right)
$$

Generation or recombination of carriers occurs also through phonon transitions in the presence of traps within the forbidden gap of the semiconductor. This is essentially a two step process modeled by the SRH mechanism as,

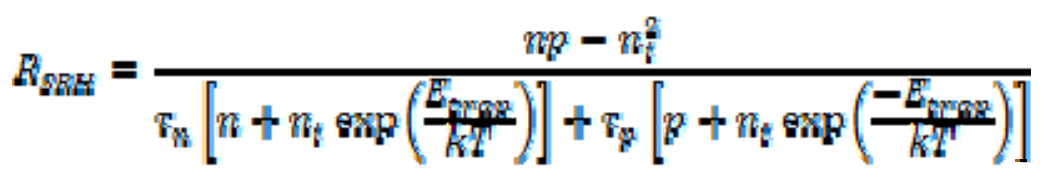


where $R_{S R H}$ is the net SRH recombination rate, $E_{\text {trap }}$ the difference between the trap energy level and the intrinsic Fermi level, $k$ the Boltzmann constant and $T$ the lattice temperature in degrees Kelvin. Traps are included to incorporate the effects of carrier capture and release in the bulk. Trap-assisted tunneling is modeled by including appropriate enhancement factors $\left(\gamma_{n, p}\right)$ to modify the trap lifetimes used in equation (4). These enhancement factors modify the lifetimes so that they include the effects of phonon-assisted tunneling on the emission of electrons and holes from a trap as given in equation (5),

$y=\frac{\Delta E_{n, p}}{k T} \int_{0}^{1} \exp \left(\frac{\Delta E_{n, p}}{k T} u-K_{n, p} u^{8 / 2}\right) d u$

where $u$ is the integration variable, $\Delta E_{n, p}$ is the energy range in which tunneling can occur and $K_{n, p}$ is defined as,

$K_{m p}=\frac{6 \pi \sqrt{2 m_{n q} \Delta E_{n q}^{a}}}{3 q h E}$

where $m_{n, p}$ is the effective tunneling mass, $h$ is Plank's constant and $E$ is the magnitude of the electric field. Surface recombination is also implemented in a similar fashion where the carrier lifetimes are modified using equation (7),

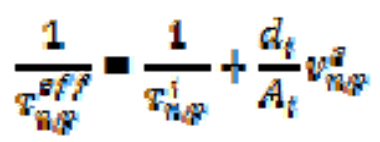

where $\tau^{\text {eff }}$ is the effective lifetime, $\tau^{i}$ is the bulk lifetime calculated at node $i$ at the interface, $d_{i}$ and $A_{i}$ are the length and the area of the interface at node $i$ and $v^{s}$ is the surface recombination velocity. This modification of the lifetime values are carried out only at the semiconductorinsulator interface nodes. Interface traps are introduced to further enhance surface recombination and trap-assisted tunneling at the semiconductor surface.

Under sufficient electric field band to band tunneling can be a significant source of current in a reverse biased diode especially in narrow bandgap LWIR materials. Carrier generation through band to band tunneling is modeled using equation (8),

$$
G_{\text {DgT }}=\sigma E^{\gamma} \exp \left(\frac{\beta}{E^{\prime}}\right)
$$

where $\alpha$ and $\beta$ are constants which depend on semiconductor bandgap and effective tunneling mass while $\gamma$ is a constant generally equal to two. The effect of the shunt resistance is simulated in the MIXEDMODE platform of ATLAS, where it is included as a resistance in parallel to the device and is simulated simultaneously in a SPICE model. 


\section{Choice of Parameter Values}

Successful implementation of the specified physical models requires specification of precise parameter values. One of the main limitations of SLS simulation is the lack of the same. Being a relatively novel material, material properties are not readily available in the literature. Moreover, it is almost impossible to generate an exhaustive list of parameter values since the material properties might change with different superlattice designs. The bandgap of the LWIR intrinsic region was determined from experimentally measured cut-off wavelength of $\sim 10 \mu \mathrm{m}$ [12]. Fourteen band k.p simulations were used to calculate the bandgap of the top layer and also the effective electron and hole masses for the superlattice structures. Hole [14] and electron [15] mobility, SRH lifetimes [16], radiative [16] and Auger [2] rates were determined following existing literature. Electron affinities and permittivity of superlattice structures were calculated as a weighted average of InAs and GaSb bulk values, which is good as a first degree approximation. The effective tunneling mass for trap tunneling was included as a mean value calculated from effective electron and hole masses [17]. Bulk trap density of $2.1 \times 10^{16} \mathrm{~cm}^{-3}$ near midgap was included to account for the currentvoltage characteristics near intermediate biases as used in previous analytical fittings [15]. Generally the same tunneling mass is used in analytical expressions for band to band and trap-assisted tunneling. However, a higher value of tunneling mass was used for band to band tunneling, the reasons for which will be discussed in the next section. Surface properties, namely surface recombination velocities, interface traps at the semiconductor-dielectric interface and shunt resistance values were used as fitting parameters to match the simulated results with the experimental ones since surface parameters vary with different passivants. The parameter values used for the LWIR region are summarized in table I.

\section{Results and Discussion}

Figure 2 shows the simulated carrier concentrations in the different regions under equilibrium without any incident radiation. The majority carrier concentration values are $2 \times 10^{18} \mathrm{~cm}^{-3}\left(\mathrm{P}^{+}\right), 4 \times 10^{18} \mathrm{~cm}^{-3}\left(\mathrm{n}^{+}\right)$and $4 \times 10^{16} \mathrm{~cm}^{-3}\left(\mathrm{n}^{-}\right)$matching with those used in the experiment. The existence of an abrupt P-n heterojunction and an $n-n^{+}$homojunction is apparent in the illustration. Uniform doping is a valid assumption for epitaxial growth using MBE. These equilibrium values are important for calculating generation-recombination rates under various non-equilibrium conditions.

Position dependent electric field values within the device are illustrated in figure 3. The electric field profiles exhibit triangular shapes with maximum values of $3 \times 10^{4} \mathrm{~V} / \mathrm{cm}$ and $4 \times 10^{4} \mathrm{~V} / \mathrm{cm}$ at the hetero and the homojunction respectively. The depletion width associated with the heterojunction is wider and is expected to increase with increasing reverse bias. However, the electric field at the homojunction is higher at equilibrium and remains significant at slightly higher biases as well.

Initial simulations using the calculated tunneling mass yielded absurdly high current densities. The issue was investigated and the problem was identified as excessive tunneling 
through the heterojunction. As with any other carrier process, tunneling demands conservation of energy and momentum. The conservation of momentum in band-to-band tunneling is relatively difficult in a heterojunction compared to a homojunction due to different effective masses and band structures in general. However, high tunneling currents in the initial simulation results indicated disregarding of such heterojunction restrictions during simulation. The values of the constants $\alpha$ and $\beta$ in equation (8) hence were changed to $6 \times 10^{16}$ and $7 \times 10^{5} \mathrm{Vcm}^{-1}$ corresponding to higher effective tunneling masses to nullify the apparent limitation of ATLAS. The originally calculated effective tunneling mass was used without any change for trap-assisted tunneling since conservation of momentum is not a critical issue for the mechanism.

The current-voltage characteristics for different passivants were successfully fitted to the experimental curves as shown in figure 4. Root mean square errors for the fittings were calculated to be $0.41,0.27,0.65$ and 0.39 for unpassivated and $\mathrm{SiO}_{2}, \mathrm{Si}_{\mathrm{x}} \mathrm{N}_{\mathrm{y}}$ and $\mathrm{ZnS}$ passivation. All bulk properties were kept unchanged while surface related properties were used as fitting parameters for different passivants. Surface recombination velocity and shunt leakage resistance affect the current at lower biases. Currents at higher biases are dominated by band to band tunneling or enhanced trap-assisted tunneling due to the presence of traps at the semiconductor-insulator interface.

$\mathrm{ZnS}$ passivated devices exhibit negligible shunt leakage path. On the contrary, $\mathrm{SiO}_{2}$ passivated current-voltage characteristic is solely influenced by the shunt resistance with a value of $0.56 \Omega-\mathrm{cm}^{2}$. Shunt resistance values extracted for unpassivated and $\mathrm{Si}_{\mathrm{x}} \mathrm{N}_{\mathrm{y}}$ passivation are $0.52 \Omega-\mathrm{cm}^{2}$ and $55 \Omega-\mathrm{cm}^{2}$. The shunt resistance values for unpassivated and silicon dioxide passivated devices match closely to experimentally measured $\mathrm{R}_{0} \mathrm{~A}$ values $\left(0.66 \Omega-\mathrm{cm}^{2}\right.$ for unpassivated and $0.72 \Omega-\mathrm{cm}^{2}$ for $\mathrm{SiO}_{2}$ passivated) indicating strong influence of surface leakage paths. Electron and hole surface recombination velocities for unpassivated, $\mathrm{Si}_{\mathrm{x}} \mathrm{N}_{\mathrm{y}}$ passivated and $\mathrm{ZnS}$ passivated devices are fitted as $10^{5} \mathrm{~cm} / \mathrm{s}$ and $10^{7}$ $\mathrm{cm} / \mathrm{s}, 10^{3} \mathrm{~cm} / \mathrm{s}$ and $10^{5} \mathrm{~cm} / \mathrm{s}, 10^{2} \mathrm{~cm} / \mathrm{s}$ and $10^{3} \mathrm{~cm} / \mathrm{s}$. A similar trend is apparent in interface trap densities as well where $\mathrm{ZnS}$ passivated devices exhibit least interface trap density of $10^{10}$ $\mathrm{cm}^{-2}$ while those for unpassivated and $\mathrm{Si}_{\mathrm{x}} \mathrm{N}_{\mathrm{y}}$ passivated devices are $10^{15} \mathrm{~cm}^{-2}$ and $8.5 \times 10^{14}$ $\mathrm{cm}^{-2}$. The fitted surface parameters are summarized in table II. Note that only the shunt resistance is reported for $\mathrm{SiO}_{2}$ passivation. The origin of the shunt resistance is most likely the presence of excessive fixed charges inside the passivation layer. Due to the image charges created at the surface, the semiconductor at the interface is never depleted and provides a continuous path for the current to flow. This does not imply that $\mathrm{SiO}_{2}$ passivation does not have surface recombination or interface traps; however, the shunt path is so dominant that those effects are masked.

Estimated surface recombination velocity values for $\mathrm{ZnS}$ passivation with prior $\left(\mathrm{NH}_{4}\right)_{2} \mathrm{~S}$ treatment are lower than those experimentally measured earlier in previous studies $\left(10^{3} \mathrm{~cm} / \mathrm{s}\right.$ in ref. [18], $4 \times 10^{5} \mathrm{~cm} / \mathrm{s}$ in ref. [19]. These studies involved $\left(\mathrm{NH}_{4}\right)_{2} \mathrm{~S}$ treatments but 
no capping layer, which might have resulted in higher values of surface recombination velocity. Moreover, in these experimental studies all surface effects are lumped in the form of surface recombination velocity; whereas in the current modeling approach interface traps and shunt resistances are included separately. Lumping all surface effects in the form of surface recombination velocity works to model $\mathrm{R}_{0} \mathrm{~A}$ values or measurement done close to zero bias in general, as performed in these studies. However, it cannot account for currentvoltage characteristics at higher biases where trap-assisted tunneling plays a more vital role. Modeling the surface effects separately reduces the surface recombination velocity values as used in the current simulation model.

A separate discussion is relevant to summarize the limitations of the ATLAS framework encountered in the process. While the tool is tried and tested for standard materials working at room temperature, there is a lack of robustness for simulations done at low temperatures in general. The numerical analysis failed to converge while simulating a n$\mathrm{N}$ heterojunction. Usage of thermionic emission yielded absurdly high current values. Bandto-band tunneling at the P-n heterojunction also rendered absurdly high current densities, the effect of which was artificially eliminated by fitting a higher effective tunneling mass. It is also mentioned already that ATLAS cannot handle the superlattices directly. It is defined as a bulk where the parameters are fed from other quantum mechanical calculations.

\section{Conclusions}

In this paper, abrupt heterojunction LWIR photodiodes based on InAs/GaSb superlattices with different surface passivants were modeled and simulated using SILVACO ATLAS. Since surface effects significantly affect SLS diode performance the surface effects were modeled along with the bulk mechanisms. The simulated results were fitted to previous experimental data and surface parameters were extracted for different passivation techniques. $\mathrm{SiO}_{2}$ passivated diode performance was found to be limited by a strong shunt leakage path with a resistance times area value of $0.56 \Omega-\mathrm{cm}^{2}$. The other diodes were affected by a combination of shunt resistance, interface trap density and surface recombination velocity. While shunt resistance and surface recombination were observed to be the dominant current mechanisms at lower biases, band-to-band and trap-assisted tunneling dominated intermediate and higher biases. Shunt resistance values for unpassivated and $\mathrm{Si}_{\mathrm{x}} \mathrm{N}_{\mathrm{y}}$ passivated diodes were extracted to be $0.52 \Omega-\mathrm{cm}^{2}$ and $55 \Omega-\mathrm{cm}^{2}$. The fitting also revealed electron and hole surface recombination velocities for unpassivated, $\mathrm{Si}_{\mathrm{x}} \mathrm{N}_{\mathrm{y}}$ passivated and $\mathrm{ZnS}$ passivated devices as $10^{5} \mathrm{~cm} / \mathrm{s}$ and $10^{7} \mathrm{~cm} / \mathrm{s}, 10^{3} \mathrm{~cm} / \mathrm{s}$ and $10^{5} \mathrm{~cm} / \mathrm{s}, 10^{2} \mathrm{~cm} / \mathrm{s}$ and $10^{3} \mathrm{~cm} / \mathrm{s}$. A similar trend was apparent in interface trap densities as well where $\mathrm{ZnS}$ passivated devices exhibited least interface trap density of $10^{10} \mathrm{~cm}^{-2}$ while those for unpassivated and $\mathrm{Si}_{\mathrm{X}} \mathrm{N}_{\mathrm{y}}$ passivated devices were $10^{15} \mathrm{~cm}^{-2}$ and $8.5 \times 10^{14} \mathrm{~cm}^{-2}$. SILVACO ATLAS provides a robust numerical analysis platform with numerous choices for physical models and flexibility with parameter specification, required for accurate simulation of SLS diodes. However, a few limitations were observed during low temperature simulations nonetheless, which were circumvented through adjustment in device structure and parameter values. 


\section{References}

[1] G. Sai-Halasz, R. Tsu, L. Esaki, A new semiconductor superlattice, Appl. Phys. Lett. 30 (1971) 651-653.

[2] C. H. Grein, P. M. Young, H. Ehrenreich, Minority carrier lifetimes in ideal InGaSb/InAs superlattices, Appl. Phys. Lett. 61 (1992) 2905-2907.

[3] K. Banerjee, S. Ghosh, S. Mallick, E. Plis, S. Krishna, C. Grein, Midwave infrared InAs/GaSb strained layer superlattice hole avalanche photodiode, Appl. Phys. Lett. 94 (2009) 201107-201109.

[4] S. Mallick, K. Banerjee, J.B. Rodriguez, S. Krishna, S. Ghosh, Ultralow noise midwave infrared InAs-GaSb strain layer superlattice avalanche photodiode, Appl. Phys. Lett. 91 (2007) 241111-241113.

[5] D.L. Smith, C. Mailhiot, Proposal for strained type II superlattice infrared detectors, J. Appl. Phys. 62 (1987) 2545-2548.

[6] Y. Choi, K. Asano, N. Lindert, V. Subramanian, T. King, J. Bokor, C. Hu, Ultra-thin body SOI MOSFET for deep-sub-tenth micron era, IEDM Technical Digest International Electron Devices Meeting (1999) 919-921.

[7] S. Michael, A novel approach for the modeling of advanced photovoltaic devices using the SILVACO/ATLAS virtual wafer fabrication tools, Sol. Energ. Mat. Sol. C. 87 (2005) 771-784.

[8] H.T Lim, F. Udrea, D.M. Garner, W.I. Milne, Modelling of self-heating effect in thin SOI and Partial SOI LDMOS power devices, Solid State Electron. 43 (1999) 1267-1280.

[9] S. Michael, P. Michalopoulos, Application of the SILVACO/ATLAS software package in modeling and optimization of state-of-the-art photovoltaic devices, 45 th Midwest Symposium on Circuits and Systems (2002) II-651-II-654.

[10] S.K. Mandal, S. Chakraborty, C.K. Maiti, Ge-channel p-MOSFETs with $\mathrm{ZrO}_{2}$ gate dielectrics, Microelectron. Eng. 81 (2005) 206-211.

[11] P.R. Palmer, E. Santi, J.L. Hudgins, X. Kang, J.C. Joyce, P. Y. Eng, Circuit simulator models for the diode and IGBT with full temperature dependent features, IEEE T. Power Electr. 18 (2003) 1220-1229. 
[12] K. Banerjee, S. Ghosh, S.Mallick, E.Plis, S.Krishna, Electrical Characterization of Different Passivation Treatments for Long-Wave Infrared InAs/GaSb Strained Layer Superlattice Photodiodes, J. Elec. Mat. 38 (2009) 1944-1947.

[13] Silvaco ATLAS Users Manual https://dynamic.silvaco.com/dynamicweb/jsp/downloads/DownloadManualsAction.do?req=S ilen-manuals\&name $=$ atlas

[14] C. Cervera, J.P. Perez, R. Chaghi, J.B. Rodriguez, P. Christol, L. Konczewicz, S. Contreras, Transport measurements on InAs/GaSb superlattice structures for mid-infrared photodiode, Journal of Physics: Conference Series 193 (2009) 012030-012033.

[15] Q. K. Yang, F. Fuchs, J. Schmitz, W. Pletschen, Investigation of trap-assisted tunneling current in InAs/(GaIn)Sb superlattice long-wavelength photodiodes, Appl. Phys. Lett. 81 (2002) 4757-4759.

[16] D. Donetsky, S. P. Svensson, L. E. Vorobjev, G. Belenky, Carrier lifetime measurements in short-period InAs/GaSb strained-layer superlattice structures, Appl. Phys. Lett. 95 (2009) 212104-212106.

[17] M. Ito, T. Kaneda, K. Nakajima, Y. Toyama, H. Ando, Tunneling currents in $\mathrm{In}_{0.53} \mathrm{Ga}_{0.47} \mathrm{As}$ homojunction diodes and design of $\mathrm{InGaAs} / \mathrm{InP}$ hetero-structure avalanche photodiodes, Solid State Electron. 24 (1981) 421-424.

[18] S. L. Chuang, E. Aifer, E. M. Jackson J. V. Li, Surface recombination velocity reduction in type-II InAs/GaSb superlattice photodiodes due to ammonium sulfide passivation, Appl. Phys. Lett. 90 (2007) 223503-223505.

[19] E. Plis, S. J. Lee, Z. Zhu, A. Amtout, S. Krishna, InAs/GaSb Superlattice Detectors Operating at Room Temperature, IEEE J. Sel. Top. Quant. 12 (2006) 1269-1274. 


\section{Figure Captions}

Figure 1 Simulated device structure $(1 \mu \mathrm{m} \times 1 \mu \mathrm{m}$ cross-section unlike $400 \mu \mathrm{m}$ diameter circular cross-section of experimental devices)

Figure 2 Simulated carrier concentration under equilibrium

Figure 3 Simulated electric field under equilibrium

Figure 4 Current-voltage characteristics of unpassivated LWIR SLS photodiodes and those passivated by silicon dioxide, silicon nitride and zinc sulfide as simulated using Silvaco ATLAS compared against experimental curves 
Table I Parameter values used for LWIR superlattice in ATLAS simulation

\begin{tabular}{cc}
\hline Parameter & Values \\
\hline Bandgap & $0.124 \mathrm{eV}$ \\
Permittivity & 14.985 \\
Electron affinity & $4.29 \mathrm{eV}$ \\
Electron effective mass & $0.023 \mathrm{~m}_{0}$ \\
Hole effective mass & $0.104 \mathrm{~m}_{0}$ \\
Electron mobility [15] & $1000 \mathrm{~cm}^{2} / \mathrm{V}-\mathrm{s}$ \\
Hole mobility [14] & $270 \mathrm{~cm}^{2} / \mathrm{V}-\mathrm{s}$ \\
SRH lifetime [16] & $90 \mathrm{~ns}^{-10} \mathrm{~cm}^{3} / \mathrm{s}$ \\
Radiative recombination rate [16] & $4 \times 10^{-26} \mathrm{~cm}^{6} / \mathrm{s}$ \\
Electron Auger recombination rate [2] & $4 \times 10^{-28} \mathrm{~cm}^{6} / \mathrm{s}$ \\
Hole Auger recombination rate [2] & $4 \times 10^{2}$ \\
Effective tunneling mass & $0.0232 \mathrm{~m}_{0}$ \\
\hline
\end{tabular}


Table II Surface parameters as fitted in ATLAS simulation for different passivants of LWIR SLS

\begin{tabular}{|c|c|c|c|c|}
\hline \multirow[t]{2}{*}{ Passivant } & \multicolumn{2}{|c|}{$\begin{array}{c}\text { Surface } \\
\text { Recombination } \\
\text { Velocity } \\
(\mathrm{cm} / \mathrm{s})\end{array}$} & \multirow[t]{2}{*}{$\begin{array}{l}\text { Interface Trap Density } \\
\qquad\left(\mathrm{cm}^{-2}\right)\end{array}$} & \multirow[t]{2}{*}{$\begin{array}{c}\text { Shunt } \\
\text { Resistance } \\
\left(\Omega-\mathbf{c m}^{2}\right)\end{array}$} \\
\hline & Electron & Hole & & \\
\hline Unpassivated & $10^{5}$ & $10^{7}$ & $10^{15}$ & 0.52 \\
\hline Silicon-di-oxide & - & - & - & 0.56 \\
\hline Silicon nitride & $10^{3}$ & $10^{5}$ & $8.5 \times 10^{14}$ & 55 \\
\hline Zinc sulfide & $10^{2}$ & $10^{3}$ & $10^{10}$ & - \\
\hline
\end{tabular}

